\title{
Das Christliche Menschenbild - Orientierung in einer pluralistischen Gesellschaft ${ }^{1}$
}

\author{
von Karl Lehmann
}

Auf den ersten Blick ist die Frage, ob es überhaupt ein christliches Menschenbild gibt, wenig ergiebig. Die Antwort scheint völlig klar zu sein. Es gibt ein solches Menschenbild. Es macht eine Stärke des christlichen Glaubens aus, daß er inmitten der Sinnkrise und eines großen pluralistischen Angebots auf ein solches Menschenbild zurückgreifen kann.

Aber da gibt es andere Töne, die eine solche rasch zupackende Antwort unsicher werden lassen. Sinnschwund und Verlust prägender Leitbilder gehören zur Signatur unserer Zeit. Bei Bert Brecht heißt es in einem Chor: Es weiß seit langer Zeit/Niemand mehr, was ein Mensch ist". Wir leben zwischen zerbrochenen Normen und Vorbildern. Zeiten mit großen Leitbildern haben - so wird weiter entgegnet - oft schändlich am Menschen gehandelt und seine Freiheit mit Füßen getreten. Damit die Ideen sich in der Geschichte nicht blamieren, wurden blutige Kriege geführt und unschuldige Menschen bedenkenlos getötet. Der Anspruch auf ein "Menschenbild" scheint im tiefsten zweideutig zu sein. Ist es nicht die Diktatur des abstrakten Sollens - "So soll der Mensch sein!" - über das konkrete "Ist" jedes Einzelnen? Liegt hier nicht die Gefahr des Totalitären in unmittelbarer Nähe? "Bilder" vom Menschen, und gar Vor- und Leitbilder, können mithelfen, den Mitmenschen einer "Idee" gemäß zu taxieren, meist negativ. Das "Bild" von einem anderen kann $\mathrm{zu}$ einem Fetisch, zu einem starrsinnigen "Leitbild" oder zu einem Götzenbild werden. "Wir selber sind die letzten, die sich verwandeln. Wir halten uns für den Spiegel und ahnen nur selten, wie sehr der andere seinerseits eben der Spiegel unseres Menschenbildes ist, unser Erzeugnis, unser Opfer" (Max Frisch).

Wir können nicht elementar genug ansetzen: Wollen wir manchmal nicht $\mathrm{zu}$ viel vom Menschen wissen? Es ist ja zuerst eine Frage an den

Prof. Dr. Dr. Karl Lehmann ist Bischof in Mainz und Vorsitzender der Deutschen Bischofskonferenz.

1 Vortrag beim Festakt "25 Jahre Institut zur Förderung publizistischen Nachwuchses e. V." am 14.11.1993 im Heinrich-Pesch-Haus in Ludwigshafen. 
Menschen selbst, wie er sich erfährt und versteht. Es ist gefährlich, vom Menschen reden $\mathrm{zu}$ wollen, ohne die ganze Last und Fülle seiner Erfahrungen über sich selbst einzubeziehen; nicht bloß die großen Stunden des Glücks und der Liebe, die dunkle Zeit des zermarternden Schmerzes und des einsamen Sterbens, sondern auch das BestialischDämonische in ihm, die öde Langeweile und Banalität, der blinde Zufall im Leben, die vielen raffinierten Lügen und Enttäuschungen, die Habgier, das Menschenunmögliche in vielen Dingen. Nichts davon darf ausgelassen oder vorschnell übergangen werden, wenn der Theologe nach dem Menschen fragt. Sieht man alles ,nur' mit der vermeintlich geschichtsenthobenen Optik des Auges Gottes selbst, dann hat man schon für ein bestimmtes Menschenbild optiert: Das Zeitliche und Leibliche des Menschen stammt zwar von Gott, aber es bleibt im letzten in der Sphäre des Nichtigen. Daraus wird ersichtlich, daß man höchst vorsichtig sein muß, im Umkreis des Menschen etwas als, unwesentlich' und ,un-menschlich, anzusehen. Auch in den alltäglichen Dingen des Schlafens, des Stehens und Gehens, des Grüßens und des Sichdahintreibenlassens, verbergen sich tiefe und oft ungeahnte Einblicke in das, was der Mensch ist.

Darum ein erster Satz: Nur wenn die Theologie den Menschen in allen seinen Erfahrungen zu Wort kommen läßt und zur Sprache bringt, kann sie wissen, wer und was er ist.

Vieles weiß die Theologie über den Menschen nicht von Gott oder von dem im Offenbarungsgeschehen gewonnenen Selbstverständnis, sondern von den menschlichen, endlichen und darum auch überholbaren Aussagen des "homo sapiens" über sich selbst. Schon weil die Theologie Gottes Wahrheit über den Menschen in dessen eigener Sprache sagen muß, entrinnt er sich nicht. Überall nimmt die Bibel solche Selbsterkenntnis mit auf. Wenn die frühchristliche Theologie z. B. vom endzeitlichen Schicksal des Menschen spricht, greift sie in der Rede von der Läuterung auf platonisches Gedankengut zurück. Wir sehen heute, wie verschieden die Griechen und die Bibel vom menschlichen "Leib" sprechen und wie verschieden das Wort "Seele" gebraucht wird. Warum kann das Alte Testament durch eine lange Zeit hindurch von Gott und von der Errettung des Menschen reden, ohne unmittelbar von einem "Jenseits" für den Menschen Zeugnis abzulegen?

Wenn schon in der Offenbarungszeit das Menschenverständnis sich so sehr im "Wort Gottes" selbst bekundet, um wieviel mehr, wenn es in nachbiblischer Zeit um das "Menschenbild" geht. Der Glaube bezieht sich auf die ganze Wirklichkeit des Menschen. Deshalb muß er die Aussagen des Menschen über sich aufgreifen und z. B. nach seiner "Natur" fragen. Aber wir wissen heute, daß die unveränderliche "Natur" des Menschen eine äußerste Anstrengung des Denkens erfordert, immer wieder neu erfragt werden muß und daß es unendlich schwer bleibt, durch alle geschichtlichen Ablagerungen und wechselnden Interpretamente hindurch auf so etwas wie ein stets gleichbleiben- 
des Wesen zu kommen. Denken wir an die Auseinandersetzungen über die "Natur" des Menschen, dann sehen wir, daß bisher überzeitlich verstandene Deutungselemente im Naturbegriff einem ganz bestimmten Weltbild entstammen, z. B. dem der Stoa. Manches, was als "Naturrecht" galt, erweist sich nachträglich als ganz selbstverständlich angenommenes römisches Recht, das Jahrhunderte hindurch tatsächlich zu einer festen „zweiten Natur" geworden war.

Auch heute ist unser Menschenbild durch spezifische Entdeckungen der Wissenschaften und durch Erkenntnisse der philosophischen Reflexion geprägt: Die Psychoanalyse zeigt uns, wie tief sich in unsere Seele die verborgene Lebensgeschichte mit ihren Ereignissen und Konstellationen eingezeichnet hat; die Verhaltensforschung erinnert uns, daß wir auch biologisch von weither kommen und wie wir noch die Verhaltensschemata unserer Vorzeit als Rest einer früheren "Natur" in uns tragen und uns damit auseinandersetzen müssen. Fazit: Das theologische Menschenbild ist immer schon geschichtlichen Ausprägungen eines bestimmten Verständnisses des Menschen verpflichtet. Es gibt gar nicht das chemisch reine, theologische Bild des Menschen, denn selbst die Interpretation z. B. der Gottesebenbildlichkeit trägt ganz deutlich die Spuren einer bestimmten Zeit.

Darum ein zweiter Satz: Jedes theologische Menschenbild ist bis in seine innersten Aussagen hinein geschichtlich bestimmt, weil es in der Rezeption und Auseinandersetzung mit dem jeweiligen Selbstverständnis des Menschen entsteht und entstehen muß.

Diese Überlegungen führen zu einem weiteren Problem: Gibt es unter diesen Voraussetzungen überhaupt ein Menschenbild und erst recht ein theologisches Bild vom Menschen? Wenn wir den Begriff "Bild" in einem sehr allgemeinen Sinn nehmen, gibt es freilich umrißhafte Konturen und darin einzelne Aussagen über den Menschen. Aber gibt es das Bild des Menschen, das gleichsam sämtliche Wesenszüge in einer Gestalt in sich vereinigt? Der Ausdruck "das Menschenbild" zielt auf einen normativen Anspruch, nicht nur auf Feststellung und Deutung von Bestehendem. Es wird ein Soll aufgezeigt, nach dem sich jeder Mensch zu richten hat. Der ethische und erzieherische Aspekt, die Sorge um ein maßgebendes Bild vom Menschen, das Willkür ausschaltet und Recht ermöglicht, der im voraus zu allen partikularen Interessen erblickte Maßstab - dies alles sind hilfreiche Dimensionen, die auf die Rettung des Menschlichen zielen. Wer weiß heute jedoch schon um das ganze Bild des Menschen? Wir sind immer neu auf der Suche nach uns, nach dem, was wir sind und sein können. Dies ist mit ein Grund, warum die Menschenbilder heute vielen ideologisch vorkommen. Dies gilt erst recht für die Situation in einer pluralistischen Gesellschaft.

Nun ist diese Situation für den christlichen Glauben nicht einfach: Wenn schon das philosophische Menschenbild immer in diesem Sinne 
,offen' bleiben muß, haben dann nicht wenigstens die theologischdogmatischen Aussagen vom Menschen unverrückbare Gültigkeit? Sicher, die Botschaft Jesu Christi ist nach der Überzeugung des Christen Gottes letztes Wort zum Menschen. Es gibt kein größeres Wort als die Sendung des Sohnes und seine Selbsthingabe in Liebe für die Vielen. Ist das nicht ein letztes Bild vom Menschen? Aber so sehr es um die bleibende Kraft des Evangeliums Jesu Christi geht, so wenig darf das Werk und die Person Jesu zu dem einen ,idealen' Menschenbild emporgesteigert werden, das nur noch alle sklavisch und ohne Rücksicht auf ihre Situation zu kopieren hätten. Gott will, daß die Menschen auch in der Nachfolge Jesu Originale bleiben.

So wäre ein dritter Satz gewonnen: Die Treue zum Evangelium Jesu Christi und zur Lehre der Kirche verlangt nicht das ungeschichtliche Festhalten an abstrakten Menschenbildern. Die Nachfolge Jesu Christi und die Sendung in eine bestimmte Situation hinein zerbrechen alle jene Menschenbilder (auch theologischer Art), die nur einen vorausfabrizierten "idealen Menschen" als typische Norm gelten lassen.

Man muß sogar noch weiter gehen: Die Kirche hat sich in ihrem konkreten geschichtlichen Gang an kein einzelnes "Menschenbild" geklammert. Ein Menschenbild als reine Theorie mit einem erschöpfenden Anspruch, alle Aspekte des Menschen einzubeziehen, gibt es weder dogmatisch noch moraltheologisch. Immer wieder wurden einzelne Elemente oder Strukturen richtweisend verteidigt oder hervorgehoben, aber nie das Ganze in den Griff genommen (vgl. z. B. auch die Pastoralkonstitution "Gaudium et spes" des II. Vatikanischen Konzils, Art. 12-22). Augustinus, Pascal, Thomas von Aquin, Teilhard de Chardin - sie haben eine ziemlich verschiedene Vorstellung vom Menschen. Und was ist mit dem Menschen bei Franz von Assisi, Bernhard von Clairvaux, Don Bosco? Es gibt offenbar innerhalb der Orthodoxie zahlreiche Deutungen des christlichen Menschen. Ja, die Existenz der Heiligen - einmal auf ihren theologischen Goldgrund gesehen, nicht in den folkloristischen Verzeichnungen - bezeugt, daß das Christentum nicht die Aufstellung eines bestimmten konkreten Menschenbildes verlangt, daß es vielmehr die Absolutsetzung eines einzigen Menschenbildes gerade nicht fördert und darum auch verschiedene konkrete "Humanismen" in sich zuläßt. Selbstverständlich kann man sich über einige abstrakte formale Daten der christlichen Anthropologie einigen (Gottesebenbildlichkeit, Verantwortung der Freiheit usw.), aber diese reichen eben noch nicht aus für ein konkretes Menschenbild.

So folgt ein vierter Satz: Gerade weil der Glaube auch die Freiheit des Menschen zu ihrer eigenen Dynamik entbindet, gibt es - zahlenmäßig - kein einziges konkretes theologisches Menschenbild. Es gibt deren unendlich viele, was nicht heißt, es gäbe keine gemeinsame Struktur.

Im folgenden sei versucht, einige Richtpunkte zu entwerfen, welche die Kraft des christlichen Menschenverständnisses im Gespräch 
mit anderen Humanismen bewähren sollen. Es kann dabei nicht um Vollständigkeit gehen oder gar um die erschöpfende Behandlung der wenigen Momente, die genannt werden. Die Offnung zum kritischen Gespräch offenbart nämlich eine wichtige Einsicht, die unseren fünften Satz darstellt: Weil es - christlich gesehen - nicht ein einziges normatives, konkretes Menschenbild gibt, kann der christliche Glaube mit all jenen Humanismen in ein Gespräch kommen, die nicht selbst ein exklusives, reduziertes und insofern totalitäres Menschenbild vertreten.

Dabei gibt es freilich nicht bloß Grenzen in Einzelfragen, sondern bereits in der formalen Vorskizzierung eines Menschenbildes. Die Unverträglichkeitsgrenze zwischen der christlichen Auffassung vom Menschen und einem zuletzt menschenfeindlichen "Humanismus" scheint in folgenden Grundlinien zu liegen:

1. Man kann nicht leugnen, daß der Mensch - wegen des Überschreitenkönnens der Faktizität - sinnvoll über sich hinausfragt. Der Christ ist überzeugt, daß der Mensch dabei nicht nur auf sich selbst zurückfallen kann. Er ist ein Wesen der Transzendenz. Damit ist auch begründet, warum der Mensch ein Wesen der Freiheit ist, dem deswegen Würde, Eigenwert und Menschenrechte zukommen. Er ist nicht unentrinnbar nur an die Bedingungen des Hier und Jetzt gebunden.

2. Der Mensch ist zwar ein vielschichtiges und plurales Wesen, dennoch ist er substantiell einer, also kein Bündel verschiedener Aggregate. Wo er in dieser Pluralität (z. B. Leib - Seele, Sinnlichkeit - Geistigkeit, Tradition - Revolution) falsch vereinfacht und reduziert wird, nimmt man ihm seine wesentliche Ganzheit. Er lebt immer in dieser unaufhebbaren Spannung.

3. Wegen der Gemeinsamkeit der menschlichen Natur und seiner alle Partikularismen übergrenzenden Auszeichnung im Ganzen der Welt ist die Universalität des Menschseins absolut zu respektieren. Es gibt keine Minderung des Menschseins durch die Zugehörigkeit zu einer bestimmten Rasse, Klasse, Nation, Partei oder Religion. Implizit ist damit auch ein Minimum menschlicher Solidarität, Brüderlichkeit und Geschwisterlichkeit gegeben.

Damit kommen wir abschließend zu einem wichtigen Kriterium als sechsten Satz: Die drei Gesichtspunkte der Transzendenz, Ganzheit und Universalität der menschlichen Natur sind ein Kriterium dafür, ob ein Menschenbild grundsätzlich sich im Bereich des christlichen Glaubens ansiedeln oder wenigstens mit diesem in ein fruchtbares Gespräch kommen kann.

Diese Grundlinien sind so etwas wie dem Glauben vorauslaufende, aber doch in ihm beschlossene Kriterien für jedes theologische Menschenbild. Von diesen Überlegungen her sollen nun einige konkrete 
Richtpunkte für ein gegenwärtiges Menschenbild herausgestellt werden. Es ist deutlich geworden, daß ein solches Bild

1. keineswegs "erschöpfend“ sein kann,

2. sich von den zeitgenössischen Anschauungen über den Menschen nicht völlig und interesselos distanzieren darf und

3. situationsbezogen bleibt, sich aber nicht von der Situation allein durchgreifend bestimmen lassen darf.

Die Situation ist zwar die Herausforderung und die Angriffsspitze, aber gerade darum eben nicht auch schon die Antwort. Ohne diesen beständigen Prozeß zwischen Situation und „Evangelium" gibt es keine wirklich theologische Aussage über den Menschen.

II.

Im zweiten Teil sollen nun einige Grundpfeiler des christlichen Verständnisses vom Menschen skizziert werden. Ich möchte drei Strukturelemente nennen und erläutern: Transzendenz und Gott, der Mensch als Person, Jesus Christus als der Mensch.

\section{Transzendenz und Gott}

Weil man nicht elementar genug ansetzen kann, darf man erste Schritte im Vorletzten nicht geringschätzen: Der Mensch, der sich nicht einfach einfügt in die "Realitäten " und vor ihnen nicht schlechthin kapituliert, das Vorfindbare nicht als letzten Maßstab nimmt, macht eine mindestens partielle Erfahrung seiner Freiheit. Nüchternheit besteht nicht im Sichbeugen unter die "Macht der Tatsachen". Das Nichtaufgehen im Faktischen, Manipulierbaren und Empirischen ist die Voraussetzung und der Anfang aller Transzendenzbewegung. Der Mensch erfährt sich in solcher Selbsterkenntnis und Entscheidung für andere Möglichkeiten in seinem Bedingtsein: Er erkennt die Herausforderung durch ein Größeres als er selbst. Ein solches Transzendieren kann verschiedene konkrete Namen erhalten: "Fortschritt" , "bessere Welt", "Gerechtigkeit", "Einheit", „Brüderlichkeit", „Freiheit" , "Gemeinschaft", „Hoffnung“. Freilich zerbrechen diese Leitbilder wieder oft, weil sie sich z. B. als Gerechtigkeit nur für eine Gruppe, Freiheit nur einer Elite usw. erweisen. Sie sind selbst Bedingungen unterworfen. Und viele solcher Programme zeigen, daß sie die Steigbügel der nun Herrschenden waren - und sonst nichts. Im Vorgang des Transzendierens liegt die Möglichkeit der Illusion, des Getäuschtwerdens durch den schönen Schein, der Überrumpelung durch fremde Interessen - soll man sich die "bessere Welt" also überhaupt "vorstellen“, d. h. imaginativ entwerfen? Ist nicht alles nur ein Traum, ein Wunschbild, ein unerreichbares und falsch vertröstendes Ideal? Oder ist es besser, an eine ideale Gerechtigkeit "an sich" und an ein der Verwirklichung unerreichbares Ideal 
der Güte zu denken? Oder gar an eine konkrete Utopie, eine "Heimat", die jeder wünscht und sucht und niemand hat?

Diese Fragen sind schwer zu beantworten. Vielleicht reicht auch eine rein rational vorgehende Schlußfolgerung am Ende nicht aus, ohne daß deswegen die "Lösung" schon irrational zu nennen wäre. Es zeigt sich jedenfalls der menschlichen Erfahrung, daß es diesen Bereich des zuerst nur negativ Anzunähernden (nämlich zu sagen, was es nicht ist ...), Unplanbaren, zunächst „leeren“ Unendlichen und Unsagbaren gibt: er ist "jenseits" aller unmittelbar planbarer und habbarer Ziele. Und dennoch gibt es für seltene Augenblicke der Erfahrung diesen abgründigen Grund reiner Freiheit, dem sich das Glück, das Leben, die Liebe und das Gute bis ins Letzte verdanken. Die Menschen und die Christen in besonderen nennen dieses unfaßbare Geheimnis von Welt und Mensch beim Namen und heißen es "Gott". Für den Glauben ist dieser nicht nur der immer fernbleibende, immer nur asymptotisch angezielte Fluchtpunkt des Daseins; er ist nicht nur aus der Ferne aufrufender und bewegender Gott. Freilich ist er auch schon als solcher derjenige, der das Leben des Menschen erhellt, der den Lohn des Guten für bitter erlittenes Unrecht verheißt, der immer wieder neue Impulse der Liebe und der Hoffnung setzt. Aber noch mehr: Er wird als ein personal begegnendes Gegenüber der Geschichte erfahren, der handelt und sich um die Sorgen und das Elend des Menschen kümmert. „Ich bin der, der ich immer für euch da sein werde" Zukunft des Menschen, ja er wird als solcher, indem er sich in dieser Zuwendung dem Menschen schenkt, zum "Inhalt" des menschlichen Lebens. Hier gibt es z. B. reine Vergebung, die nicht wieder nachträgt, sondern - wie das Wunder der Schöpfung - alles neu macht wie am Anfang.

\section{Der Mensch als Person}

Der Mensch lernt, in der Begegnung mit Gott die verschiedenen Situationen der Bedrohung und des Unheils zu bestehen. Dieser Gott bedeutet vor allem Rettung. Er bewahrt vor Knechtschaft. Sich ganz auf ihn und seinen Weg einzulassen, heißt Glauben. Dies heißt zugleich, die ständigen Götzenbilder, die sich immer wieder dazwischenschieben, zu durchschauen und zu entmächtigen. Es gibt einen Weg zu Gott nur durch die Kritik der Idole und der ständig wiederkehrenden Versuchung, ihn mit anderem als ihn selbst zu verwechseln. Das Bilderverbot bleibt die Erinnerung dafür, daß es stets dieses Auszugs aus dem Gewohnten und Vertrauten bedarf. „Du sollst Dir kein geschnitztes Bild machen!" Die Götter und Dämonen verschwinden aus einer Welt, die als Schöpfung des in der Geschichte wirkenden Gottes angenommen wird.

Unter den Geschöpfen nimmt der Mensch eine einzigartige Sonderstellung ein. Von allen Geschöpfen ist allein der Mensch zum 
Bild Gottes geschaffen und bestimmt. In diesem Ebenbild kann Gott seinen Partner, sein Echo finden. Im Bild des Menschen will Gott auf Erden gegenwärtig sein, und sein Ebenbild soll in seinem Namen handeln. Der Mensch ist nicht nur Schöpfung Gottes, sondern "Bild" Gottes: Er ist ein Spiegel dessen, der die Freilheit ist. Die unendliche Ferne des Schöpfers von seinem Geschöpf findet sich im Menschen wieder als die Freiheit gegenüber allen endlichen Dingen und Verhältnissen. Der Glaube an diese Bestimmung verleiht eine eigene Würde. Diese Würde hat einen eminent kritischen Sinn: Sie verbietet jede Vergottung eines Herrschers, Führers, Genies, einer Nation, einer Rasse, einer Gesellschaft. Nicht weniger gilt dies für die Politik. Weil der Mensch in diesen Sinne das Ebenbild Gottes ist, darf er auch nicht zum Mittel für einen Zweck gemacht werden. In diesem „Bild" soll man Gott selbst begegnen. Das Leben des Menschen ist darum in dieser Hinsicht letzter Zweck. In seiner Erkenntnis und in seiner Entscheidungsmöglichkeit liegt ein letzter unverfügbarer Grund, der zwar in alle Handlungen eingeht, aber dennoch nie verfügbar wird. In der Freiheit zum Guten wurzelt die Personalität des Menschen. Und da Gott selbst schöpferischer Partner für alle Menschen ist, hat er jeden auch beim Namen gerufen.

Man darf jedoch diese Stellung des Menschen als Mitte und Krone der Schöpfung, die man nicht übersehen kann, nicht falsch verstehen. Der Mensch der Bibel ist ein Mitgeschöpf der Tiere und der Engel. Die Schöpfung ist weiter als der Mensch. Er selbst steht in ihr als einer, der die Erde zugleich "bebaut und bewahrt" (Gen 2,15): er gestaltet sie um und entwirft schöpferisch Neues aus ihr, er ist jedoch zugleich auf sie angewiesen und muß sie hegen und pflegen. Beides die Zugehörigkeit zur "Natur" und seine Überlegenheit ihr gegenüber spiegelt sich wider in der Kultur. In diesem Sinne ist der Mensch als Person radikal weltlich und geschichtlich, ohne daß er deshalb in dieser Zeit aufgehen müßte. Aber es kommt darin wenigstens im Ansatz zum Ausdruck, wie er sein irdisches "Haus" bestellen muß; daß er nicht seine Lebensbedingungen unterminieren und nicht realisieren darf, was seine einmalige Menschenwürde verletzt und gefährdet.

\section{Jesus Christus, der Mensch schlechthin}

Jesus Christus zeigt uns in besonderer Weise, wer und was der Mensch ist. In ihm sehen wir die letzte Größe und Würde des Menschen, daß er nicht nur gottebenbildlich ist, sondern daß er in der Wurzel seines Wesens sogar die Möglichkeit hat, Gottes eigenes Wesen zu empfangen, "capax Dei" ist. In ihm wird aber nicht bloß der wahre Gott, sondern auch der wahre Mensch offenbar. "Ecce homo" - Seht, welch ein Mensch! Seht, was der Mensch ist! Wie aber wird in ihm das Menschliche offenbar?

Von Anfang bis zum Ende seiner öffentlichen Tätigkeit kommen 
Menschen zu ihm mit allen möglichen Bitten, Fehlern, Sünden und Krankheiten. Er hat nicht ein neues "Menschenbild“ gepredigt, sondern er hat das Evangelium vom kommenden Gottesreich den Armen gebracht, Kranke geheilt, Randsiedlern der damaligen Gesellschaft Heimat gewährt und Trauernde, Weinende, Hungernde selig gepriesen. Es sind Menschen, die gar nicht mehr beachtet werden, mit denen kein Staat und auch keine Revolution zu machen ist. Aber er hat in ihrem erbärmlichen Leben das Wort "Gott" als die einzige Befreiung zur Sprache gebracht. Es sind seine "geringsten Brüder". Gott hat die Menschen auf jene Weise angenommen, die uns Jesus offenbarte. Indem er ganz für die Menschen da ist, ohne die geringste Möglichkeit und den Willen der Einschränkung (denn er gibt sein Leben für alle!), wird er schlechthin zu dem Menschen für alle, ja zum Inbegriff der Menschheit, zum letzten Menschen, nach dem kein größerer mehr gedacht werden kann. Aber auch in dieser letzten Erfüllung des Menschenbildes wird deutlich, daß dies etwas anderes ist als ein "totaler Humanismus" obwohl Jesus ganz Mensch ist. Die Tiefendimension des Menschen ist nicht durch den Menschen oder auch die Menschheit allein auszufüllen. Dieser Mensch kann nur in dieser Weise so vorbehaltlos und radikal Mensch für andere sein, weil er zugleich in der Macht des Menschensohnes und des von Gott gesandten Messias kommt. Im Gekreuzigten kommt beides zum Höhepunkt: „Darum sagt der Glaube über den Gekreuzigten beides: Ecce homo - Sehet den Menschen in der Wirklichkeit seiner ganzen Verlassenheit. Und: Ecce deus - Sehet Gott in der Wirklichkeit seiner unendlichen Liebe, mit der er sich selbst verläßt, um der Verlassenen Gott und der sich selbst Aufgebenden Vater und der sich selbst Verklagenden Freisprecher, aber auch der sich selbst Rühmenden Richter zu sein" (J. Moltmann).

Dies alles müßte in einer philosophischen und theologischen Anthropologie ausgeführt werden. Ich wollte es jedoch bewußt einmal bei einer ersten Grundlegung belassen und nicht schon auf die vielen Einzelfragen eingehen, auf den Ursprung des Menschen und die Evolution, auf Mann und Frau, die Fundierung der Werte, Person und Gemeinschaft, Menschenwürde und Sterben. Hier sollte nur der Rahmen sichtbar werden, in dem diese Einzelthemen ausgearbeitet werden.

Was hier über das Verständnis des Menschen gesagt worden ist, bedarf der ständigen Bewährung im Disput der pluralistischen Gesellschaft. Dafür scheinen mir drei Maßstäbe und Verfahrensweisen besonders nützlich zu sein, die das Humanum und Proprium des Christlichen miteinander verbinden. Ich möchte diese dreifach-eine Bewegung kurz skizzieren:

1. Integration und Vermittlung: Dem Christlichen eignet eine große Kraft der Annahme und der Anverwandlung, die freilich zugleich auch Veränderung besagt. Alles, was partikular an Gutem und Wahrem gefunden wird, kann in einen größeren Zusammenhang eingeholt 
und heimgeholt werden. Es bleibt dann immer noch ein offenes Gefüge, da es sich nicht in der eigenen Endlichkeit verschließt. Im Prozeß von Anknüpfung und Widerspruch erneuert sich immer wieder das Ganze.

2. Impuls und Dynamik: Die Dynamik des Christlichen ergänzt nicht nur, sondern ist Ansporn und drängende Kraft zur Verwirklichung menschlicher Möglichkeiten, die oft aus Trägheit und Schwerfälligkeit bisher nicht erreicht werden konnten. Das christliche Verständnis vom Menschen ist so im gesellschaftlichen Streit ein Stimulans, um aus eingefahren Gleisen herauszukommen.

3. Schöpferisch-kritischer Einspruch: Der Glaube hat die Pflicht, gegen alle Absolutsetzungen immanenter Sozialverhältnisse, Zustände und Utopien kritisch wachsam zu sein. Die schöpferische Kraft zu einer solchen Kritik kommt der Vernunft des Glaubens letztlich aus ihrer Verankerung in der Transzendenz zu. Deswegen ist sie auch nicht nur negativ, sondern im ursprünglichen Sinne schöpferisch-kritisch.

Damit ist auch einsichtig, wie eng diese Bewegung in den drei Schritten aufeinander angewiesen ist und gleichzeitig fruchtbar sein kann.

III.

Was ich vorgetragen habe, scheint nur im ersten Augenblick nichts zu tun zu haben mit dem, was wir heute feiern. In Wirklichkeit ist jedes Kommunikationsgeschehen offen oder heimlich von den hier behandelten Grundfragen bewegt. In besonderer Weise nimmt die Publizistik an diesem Austausch teil. Dieser Austauschprozeß ist nicht beliebig, sondern braucht Vorbildung und reflektierte Erfahrung. Seit den schwierigen Anfängen der Journalistenausbildung geht es darum, Studium und praktische Ausbildung zu verbinden. Es war schon das Programm von Emil Dovifat: "Investieren Sie in den Menschen!"

Wir begehen heute die 25. Wiederkehr der Gründung des Instituts zur Förderung publizistischen Nachwuchses e. V. Wir wollen jedoch nicht vergessen, daß seit mehr als 120 Jahren immer wieder, vor allem vom Zentralkomitee der deutschen Katholiken, die Gründung von Journalistenschulen gefordert worden ist (vgl. den Düsseldorfer Katholikentag von 1869). Bernhard Hanssler hat schließlich mit Alois Schardt das Programm für die sogenannten „Bensberger Kurse“ (seit 1959) entworfen. Bald nach dem Konzil beschloß die Publizistische Kommission der Deutschen Bischofskonferenz die Einsetzung eines Unterausschusses "Förderung des publizistischen Nachwuchses". Zwei Jahre später wurde das Institut gegründet. P. Wolfgang Seibel SJ war als sein Leiter vorgesehen. Ich brauche nicht zu entscheiden, wer der eigentliche Vater des Instituts ist. Viele, die damals schon große Verdienste hatten, 
sind Gott sei Dank noch unter uns, z. B. Prof. Otto B. Roegele, Prälat Prof. Bernhard Hanssler, Weihbischof Walther Kampe; andere sind von uns gegangen wie Prälat Prof. Dr. Karl Forster, Msgr. Kochs, Weihbischof Siegel und nicht zuletzt Dr. Hans Suttner. Für ihn sollten die Ziele des Hauses im folgenden bestehen: "Schaffung und Betreuung einer Journalistenschule neuen Stils. Damit soll der auf die Dauer bedeutsamste Beitrag zur Hebung des publizistischen Niveaus der katholischen Presse geleistet werden. Ziel ist der Publizist mit abgeschlossenem Hochschulstudium eines beliebigen Faches, der es trotz seiner Fachbildung versteht, gemeinverständlich zu reden und zu schreiben und der wegen seiner Fachbildung zuverlässig publizieren kann." (Katholische Presse in Deutschland, Regensburg 1965, 43f). Später kam (seit 1976) eine Volontärausbildung hinzu, die mit der Arbeitsgemeinschaft Katholische Presse (AKP) und der Medien-Dienstleistungs-GmbH (MDG) gemeinsam veranstaltet wird. Seit 1970 haben über 700 Teilnehmer einen der drei Ausbildungsgänge (Stipendiaten, Volontäre, Theologen) absolviert. Die Übernahme des Studios Ludwigshafen (1987) und die Errichtung der Außenstelle Leipzig im Jahr 1991 haben die Ausbildungsmöglichkeiten beträchtlich erweitert und verbessert. Sehr dankbar sind wir Prof. Innerhofer, daß er nach der jahrzehntelangen, hoch anerkannten Führung durch P. Dr. Seibel SJ mit den Mitarbeitern des Instituts reibungslos und nahtlos die Sache fortführt.

Das Institut hat in diesen 25 Jahren große Wirkung entfaltet und in vieler Hinsicht Segen gebracht. Viele Redakteurinnen und Redakteure sind in säkularen Medien, oft an gewichtiger Stelle, tätig. Nicht wenige sind freie Journalisten oder auch Medienreferenten geworden. Über 100 Theologen sind nebenamtlich journalistisch aktiv. Man kann sagen, daß viele Absolventen des Instituts inzwischen in allen Medien tätig sind.

Diese Tatsache sagt viel über das Verhältnis von Kirche und Öffentlichkeit. Das Institut zeigt, daß wir nicht der Meinung sind, die Kirche müßte und dürfte nur auf ihre eigenen Einrichtungen vertrauen und würde gleichsam nur für sich selbst Nachwuchs produzieren. Es kommt künftig darauf an, in vielen, möglichst allen Medien durch überzeugte katholische Publizisten, die je an ihrem beruflichen Ort ihr selbständiges Zeugnis geben, gegenwärtig zu sein. Der Arm der Institution Kirche reicht nicht so weit wie die ausgestreckten Hände vieler überzeugter und komptetenter Christen vor Ort. „Von einem festen Standort aus argumentierend können sich katholische Journalistinnen und Journalisten einmischen, ihre Stimmen in die Fragen, die unsere Zukunft bestimmen, mit Aussicht auf Erfolg zu Gehör bringen" (Eva Maria Streier, in: Bekanntmachung. 40 Jahre GKP, Berlin 1988, 124). Gerade wenn der institutionsgebundene Einfluß schwächer wird, hängt viel davon $a b, d a ß$ zeugnisbereite und kompetente Laien die wichtigsten Vermittler christlichen und auch kirchlichen Geistes werden, zumal im politischen, ökonomischen und kulturellen Vorfeld. Dafür braucht es zwischen den Verantwortlichen in der Kirche und allen, die zu einer solchen Zeugenschaft bereit sind, ein offenes, vertrauensvolles Miteinander. 
In einer Öffentlichkeit, die oft sehr diffus und schwerfällig ist, bedarf es für diese Zeugnisbereitschaft einer fundierten Kenntnis der Fragen, die zur Debatte stehen. Darum ist eine eigene Ausbildung notwendig. Dabei sind selbstverständlich nicht nur katholische Themen gemeint (was sind diese heute eigentlich?), vielmehr sollen alle Probleme und Aufgaben unserer Gegenwart vom Standort eines wachen Katholiken aus behandelt werden. Die erwünschte Wirkung wird auf die Dauer nicht ausbleiben. Ich freue mich, daß zwischen dem Institut und der Gesellschaft Katholischer Publizisten Deutschlands ein guter Zusammenhang besteht, gerade in der jüngeren Generation, denn in unserer gesellschaftlichen Situation, wo der Einzelne auch sehr rasch isoliert ist, sind gute menschliche Kontakte, ein Kreis Gleichgesinnter und die Möglichkeit einer gezielten Fort- und Weiterbildụng sowie spirituelle Angebote eine unerläßliche Voraussetzung zum Überleben. Hier sehe ich eine wichtige Verzahnung zwischem dem "Institut" und der "Gesellschaft", sicher auch noch mit anderen Einrichtungen (wie z.B. MDG, AKP).

Ich war schon immer der Meinung, daß das "Institut" in dieser zukunftsgerichteten Struktur für die Kirche geradezu exemplarischen und modellhaften Charakter hat und weit über das Berufsfeld des Publizisten hinaus von wegweisender Bedeutung ist. Erst recht gilt dies aber für die Welt der Medien und für jede Offentlichkeitsarbeit. In diesem Sinne gilt es, die hier in 25 Jahren geleistete Arbeit eher auszubauen und zu verstärken - auch wenn die Kassen knapper werden sollten. Hier geht es um die rechte Form der Zukunftsfähigkeit des Glaubens und der Kirche.

\section{Anhang:}

Ansprache des Vorsitzenden der Deutschen Bischofskonferenz, Bischof Karl Lehmann, im Gottesdienst anläßlich der Feier "25 Jahre Institut zur Förderung publizistischen Nachwuchses e. "V." am 14.11.1993 in Ludwigshafen (Pesch-Haus)

Lesung für den Gottesdienst „Eure Worte seien immer freundlich, jedoch mit Salz gewürzt" $(\mathrm{Kol} 4,2-6)$

„Laßt nicht nach im Beten; seid dabei wachsam und dankbar! Betet auch für uns, damit Gott uns eine Tür öffnet für das Wort und wir das Geheimnis Christi predigen können, für das ich im Gefängnis bin; betet, daß ich es wieder offenbaren und verkündigen kann, wie es meine Pflicht ist. Seid weise im Umgang mit den Außenstehenden, nutzt die Zeit! Eure Worte seien immer freundlich, doch mit Salz gewürzt; denn ihr müßt jedem in der rechten Weise antworten können."

Der Brief an die Kolosser ist ein Dokument des Glaubens aus einer schon etwas späteren Zeit, in der die Christen um ihre Weltverantwortung zu wissen beginnen. Der Brief schließt mit letzten 
Mahnungen an die Christen. Leicht kann man zwei Grundaussagen unterscheiden, die jedoch wiederum aufeinander verweisen: die Solidarität des fürbittenden Gebetes und die Solidarität des verantwortlichen Dialogs gerade mit den Außenstehenden. Zur Sendung in die Welt gehört auch die Verwurzelung im Glauben, die hier mit dem Dreiklang Immerwährendes Gebet - Wachsamkeit - Dankbarkeit umschrieben wird (V 2). Der Apostel ruft die Gemeinde besonders auf zum Gebet für ihn, "damit Gott uns eine Tür öffnet für das Wort und wir das Geheimnis Christi predigen können" (V 3). Obgleich er im Gefängnis sitzt, verschließt er sich nicht in einem Ghetto, auch nicht in der Abkapselung der Verfolgung. Es kommt kein Wort der Klage über seine Lippen. Er bittet um das Gebet für die missionarische Verkündigung. Hier werden die beiden Hälften des Textes verbunden: Fürbitte und Zeugnis nach draußen. Gott möge eine Tür öffnen für das Wort (vgl. 1 Kor 16,9; 2 Kor 2,12; Apg 14,27). Gottes Wort selbst drängt in die Welt. Der Apostel möchte die Herzen vieler Menschen für das Evangelium öffnen. In der Mitte steht das Geheimnis Christi (vgl. V 3). Der ganze Text - darum paßt er so gut zu diesem Tag - spricht von der Öffentlichkeit der Verkündigung und des Zeugnisses. Durch die Verkündigung soll das Mysterium allen zugänglich werden. Es ist ein offenes Geheimnis, das Einsatz und Freimut braucht, um zu anderen Menschen zu gelangen. Hier herrscht ein anderer Ton: keine Rede von Verstockung (vgl. Mk 4,11f), keine verschlossene Türe (vgl. Lk 12,36f; $21,24 f)$.

Trotzdem ist es mit "Öffentlichkeit" noch nicht getan. Druckerschwärze und Kamera, Mikrophon und Mattscheibe, Plakate und Lautsprecher erreichen für sich noch nichts. Sie können sogar abspenstig machen. Auch muß nicht jeder predigen oder missionarisch verkündigen. Dies ist Sache des Apostels, für den es geradezu eine Pflicht ist, obgleich das Evangelium letztlich von selbst läuft.

Zum Gebet um den Segen der Mission gehört auch der weise Umgang mit den "Außenstehenden". Die Gemeinde lebt in einer Umwelt, in der sie kritisch beobachtet und beurteilt wird. In einer solchen Situation hat jeder Veranwortung auch im Lebenswandel. Ohne Weisheit, d. h. hier Klugheit und Rücksicht, gibt es keine positive Resonanz bei den Menschen "draußen“ (vgl. 1 Thess 4,12): „So sollt ihr vor denen, die nicht $\mathrm{zu}$ euch gehören, ein rechtschaffenes Leben führen und auf niemand angewiesen sein" (vgl. 1 Kor 5,12f).

Aber die indirekte Rechenschaft durch das Leben selbst genügt nicht. Der Christ soll jedoch gefaßt sein, daß er auf sein Zeugnis angesprochen wird. Er soll bereit sein, auf Fragen zu antworten und Rechenschaft abzulegen von seiner Hoffnung (vgl. 1 Petr 3,15). Er soll dabei "freundlich", Luther sagt "lieblich", sein. Jedenfalls darf das Zeugnis des Wortes nicht aufdringlich werbend noch rechthaberisch sein, freilich auch nicht feige und furchtsan. Die zeugnishafte Rede des Christen muß Qualität haben. Eloquenz und Argumentationsgeschick 
sind durchaus erwünscht. Sie soll einen gewissen Anreiz, ja Charme geben (vgl. dasselbe Wort für "Gnade" = charis). Die meisten übersetzen: in Anmut, anmutig.

"Eure Worte seien immer freundlich, doch mit Salz gewürzt" (V 6a). Das heißt auch, daß unsere Worte packend und treffsicher, ja gepfeffert und entschieden sein sollen, nicht fade und faul, langweilig und kompromißlerisch. Es darf ruhig eine "gesalzene Rede", ja aufreizend und manchmal beißend sein. Es genügt jedoch nicht, etwas Charme zu produzieren oder einen "Biß" zu haben, zu provozieren. Es ist ein eigenes Wort. Es ist nicht nur unser Wort. Gott selbst öffnet die Tür zu den Herzen der Menschen. Schon gar nicht darf man das Wort der "Außenstehenden" einfach nachplappern oder sich anpassen. Es ist keine Hilfe, dem Menschen nur nach dem Mund zu reden. Man muß im Gebet wachsam bleiben gegen alles, was angebetet wird. Was gesagt wird, muß auch vom Leben gedeckt sein. Wir dürfen die Welt nicht ernster nehmen als den Herrn. Nur wenn wir selbst in diesem Sinne "Salz der Erde" (Mt 5,13) sind, das nicht schal ist, das vielmehr würzig-frisch ist und Fäulnis beseitigt, werden wir Menschen gewinnen. So kommt es darauf an, daß wir die doch auch verborgene Kraft des Geheimnisses Christi an den Tag bringen, ja regelrecht offenbaren (vgl. V 4). Unsere Freundlichkeit soll die Freundlichkeit Gottes vermitteln (weswegen die Freundlichkeit von V 6 am Ende doch auch etwas mit der „Gnade“ Gottes zu tun hat). Ein altes jüdisches Wort sagt: „Die Welt kann nicht ohne Salz und nicht ohne Pfeffer, auch nicht olhne Gewürze sein“ (Trakt. Sopherim 15,8; Billerbeck I, 232ff).

Der Apostel ist der Meinung, daß wir nur so ,jedem in der rechten Weise antworten können " $(\mathrm{V}$ 6b). Auch dies ist nochmals wichtig: Wir sollen uns auf jeden einlassen, nicht einfach nur einen Propagandafeldzug eröffnen, sondern uns den Fragen der Menschen stellen und dann auch "jedem in der rechten Weise" antworten. Wir wissen, wie viel dies voraussetzt. Auch dies ist gut jüdisch, denn es heißt: "Wohl dem Mann, der die Worte der Thora zu eigen hat und in dessen Hand sie verwahrt werden, und der es versteht, mit ihnen am rechten Ort eine vollkommene Antwort zu geben" (Billerbeck III, $765 \mathrm{zu}$ 1 Petr 3,15).

Dafür sollen wir "die Zeit nützen“: jede Gelegenheit nützen für das rechte Wort zur rechten Zeit. Es gibt kein dringlicheres Wort, da es in dieser Zeit von niemand überholt werden kann. Also müssen wir auch unseren Ort mit unseren Möglichkeiten ausschöpfen. Dieses letzte Wort ist durchaus eschatologisch gemeint.

Es ist ein unglaublich dichter Text, der sich einem hier erschließt. Er schenkt uns eine kleine praktische und theoretische Theologie der Kommunikation des Christlichen in der Zeit. Ich brauche darum diese Wort auch nicht zu übersetzen in die Gegenwart. Jeder entdeckt seine Möglichkeiten in seiner Situation. Die Lesung aus dem Kolosserbrief ist 
wie ein Kommentar zu dem Jesuswort des heutigen Evangelientextes: "Darum fürchtet euch nicht vor ihnen! Denn nichts ist verhüllt, was nicht enthüllt wird, und nichts ist verborgen, was nicht bekannt wird. Was ich euch im Dunkeln sage, davon redet am hellen Tag, und was man euch ins Ohr flüstert, das verkündet von den Dächern" (Mt 1026f; vgl. Mk 4,22; Lk 8,17). Der Herr selbst macht uns zu Offenbarern, zu „Publizisten“: das sind Leute, die über öffentliche Angelegenheiten schreiben. Das Evangelium ist eine eminent öffentliche Angelegenheit und nicht die private Anmutung, zu der wir es oft gemacht haben und machen. Gegen diese moderne Häresie steht der Publizist, der Christ ist und sein will, mit Leib und Seele, mit Haut und Haar. Amen.

SUMMARY: The Christian view on mankind - orientation in a pluralistic society

In this text the chairman of the conference of the German bishops raises the question of the existance of a specific Christian view on mankind. In a first step he formulates in six theses the basic perspectives and aspects of a theological anthropology. He distincts, that in Christian faith a theological or philosophical view on mankind cannot exist as a mere theory with the exhausting claim to integrate all aspects of human being. For that reason Christianity is able to get in touch with all kinds of humanism, which do not advocate an exclusive, reduced and in that totalitarian anthropological approach.

In the second part of the text the author denominates some main supports of the Christian understanding of human being. He develops them by means of three thematical lines: transcendence and god, man as person, Christ as the human being. With regards to the relation between the Church and the public, the author concludingly pleads for competent catholics, who are able to hold the dialog with the pluralistic world and to work in any range of media. This is for the task to deal with any present-day problem and challenge from a position of an "alert catholic".

RÉSUMÉ: La conception chrétienne de l'homme. Orientation dans une société pluraliste

Dans ce texte, le président de la Conférence Allemande des Evêques pose la question à sacoir s'il existe une conception de l'homme purement chrétienne. Dans un premier temps, il exprime en 6 thèses les perspectives et aspects sur lesquels peuvent reposer une anthropologie théologique et il fait comprendre qu'il ne peut pas y avoir dans la croyance chrétienne, au niveau théologique ou philosophique, une conception de l'homme en tant que pure théorie ayant la prétention exhaustive de regrouper tous les aspects de l'homme. C'est la raison pour laquelle le christianisme peut entrer en discussion avec chaque humanisme qui, lui même, ne défend pas une conception de l'homme exclusive, réduite et donc totalitaire.

C'est sur cette base que l'auteur nous donne dans un deuxième temps quelques piliers importants de la compréhension chrétienne de l'homme qu'il développe en 3 thèmes: Transcendance et Dieu, 1,homme en tant que personne, Jésus Christ en tant qu'homme. Pour conclure, l,auteur plaide, en vue des rapports entre l'église et le public, pour le travail de catholiques compétents et capables de dialoguer dans tous les secteurs de l'audio-visuel, afin que les 
problèmes et les devoirs du présent soient aussi traités à partir du point de vue d'un "catholique éveillé".

RESUMEN: La visión cristiana del ser humano - Orientación en una sociedad pluralista

En este texto el Presidente de la Conferencia Episcopal alemana tematiza la pregunta de si existe un visión cristiana propia del ser humano. En un primer paso formula en 6 tesis algunos aspectos y perspectivas para la creación de una antropología teológica, poniendo en claro que en la fé cristiana no puede existir una visión teológica o filosófica del ser humano, que como teoría pura pretenda integrar en forma exaustiva todos los aspectos del ser humano. De ahí que el cristianismo pueda entablar conversaciones con todos los humanismos que no representen concepciones o visiones del ser humano exclusivas, reductivas $y$, por lo tanto, totalitarias.

Teniendo lo anterior como base el autor formula en la segunda parte algunos pilares sobre los que descansa la concepción cristiana del ser humano, desarrollándo tres aspectos: Trascendencia y Dios, el ser humano como persona, Jesucristo como ser humano. Como corolario el autor aboga, refiriéndose a la relación entre la Iglesia y la opinión pública, por que trabajen en todos los campos des los medios de comunicación católicos competentes y dispuestos al diálogo para que así todos los problemas y tareas del presente puedan ser tratados también desde el punto de vista de un "católico despierto". 